\title{
Active targeting significantly outperforms nanoparticle size in facilitating tumor specific uptake in orthotopic pancreatic cancer
}

William M. MacCuaig ${ }^{1,3}$, Benjamin L. Fouts ${ }^{1}$, Molly W McNally, 2,4, William E. Grizzles, Phillip Chuong ${ }^{6}$, Abhilash Samykutty ${ }^{1,3,4}$, Priyabrata Mukherjee ${ }^{l}$, Min Li ${ }^{1}$, Jacek B. Jasinski ${ }^{7}$, Bahareh Behkam ${ }^{8}$, Lacey R. McNally 1,2,3,4*

1 Stephenson Cancer Center, University of Oklahoma, Oklahoma City, OK, 73104, USA

2 Department of Surgery, University of Oklahoma, Oklahoma City, OK, 73104, USA

3 Department of Biomedical Engineering, University of Oklahoma, Norman, OK 73019, USA

4 Department of Cancer Biology, Wake Forest University, Winston-Salem, NC 27157, USA

5 Department of Pathology, University of Alabama at Birmingham, Birmingham, AL 35294, USA

6 Department of Surgery, University of Louisville, Louisville, KY 40202, USA

7 Conn Center Materials Characterization, University of Louisville, Louisville, KY 40202, USA

8 Department of Mechanical Engineering, Virginia Tech, Blacksburg, VA, 24061, USA

\section{Contents}

Figure S1. 3D model of V7 peptide using online software EzMol. V7 consists of 26 amino acids and is approximately $2.6 \mathrm{~nm}$ in length. The CYS residue utilized to attach the V7 peptide to the nanoparticle via SMCC is noted in red.

Figure S2 Characterization of IR780 dye within TROS. Graphs depict (A) IR780 standard curve, (B) measurement of optical density compared to IR780 dye within TROS, and (C) number of dye molecules per particle for given concentrations of IR780 in TROS....

Figure S3 Evaluation of cell viability following TROS and V7-TROS treatment. (A) No significant toxicity was observed in malignant (S2VP10 and MiaPaca-2) or non-malignant (THLE-2 and NK-2) cells at any particle size for V7 actively targeted TROS ( $\mathrm{p}>0.05)$. (B) No significant toxicity was observed in malignant (S2VP10 and MiaPaca-2) or non-malignant (THLE-2 and NK-2) cells at any particle size for passively targeted TROS $\mathrm{p}>0.05$....

Figure S4 MSOT detection of sub-millimeter PDAC tumor via V7-TMOS in vivo. Orthotopic submillimeter $(0.72 \mathrm{~mm})$ PDAC tumor detected using V7-TMOS (A) or oxy/deoxyhemoglobin (C). 
Orthotopic sub-millimeter $(0.23 \mathrm{~mm})$ PDAC tumor detected with V7-TMOS (B). The 0.23mm tumor was not detected with oxy/deoxyhemoglobin only (D). NIR fluorescence was used to detect submillimeter tumors of size $0.72 \mathrm{~mm}$ (E). NIR fluorescence was unable to detect PDAC tumors of $0.23 \mathrm{~mm}(\mathrm{~F})$. Confirmation of sub-millimeter tumor using NIR fluorescence $(\mathrm{G})$.

Figure S5 Assessment of blood retention of V7-TROS and TROS signal at timepoints of 0, 4h, 8h, and 24h post intravenous injection. Signal was measured using a region of interest analysis at a single snapshot at each timepoint in the aorta. The levels of V7-TMOS and V7-TEOS in the blood (represented by the aorta) were not statistically different ( $\mathrm{p}=0.57$ ), however both V7-TMOS and V7-TEOS were significantly higher than V7-TPOS $(\mathrm{p}=0.046)$

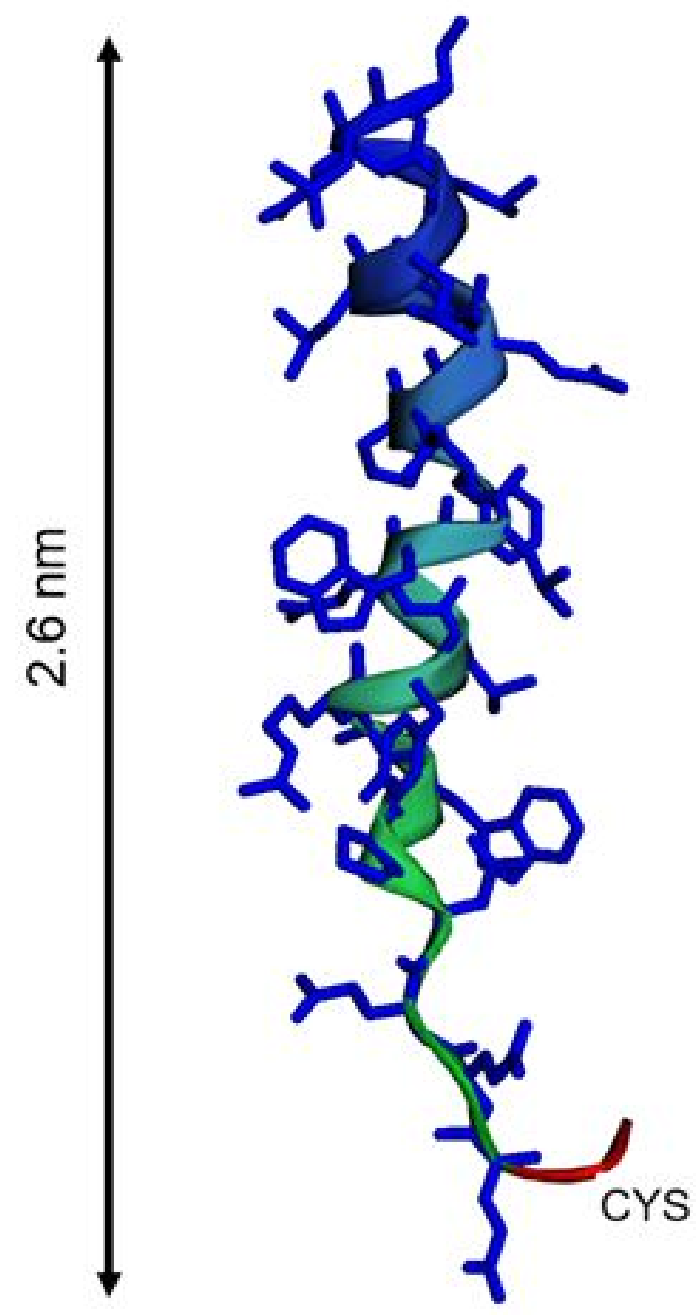


Figure S1 3D model of V7 peptide using online software EzMol. V7 consists of 26 amino acids and is approximately $2.6 \mathrm{~nm}$ in length. The CYS residue utilized to attach the V7 peptide to the nanoparticle via SMCC is noted in red.

S1
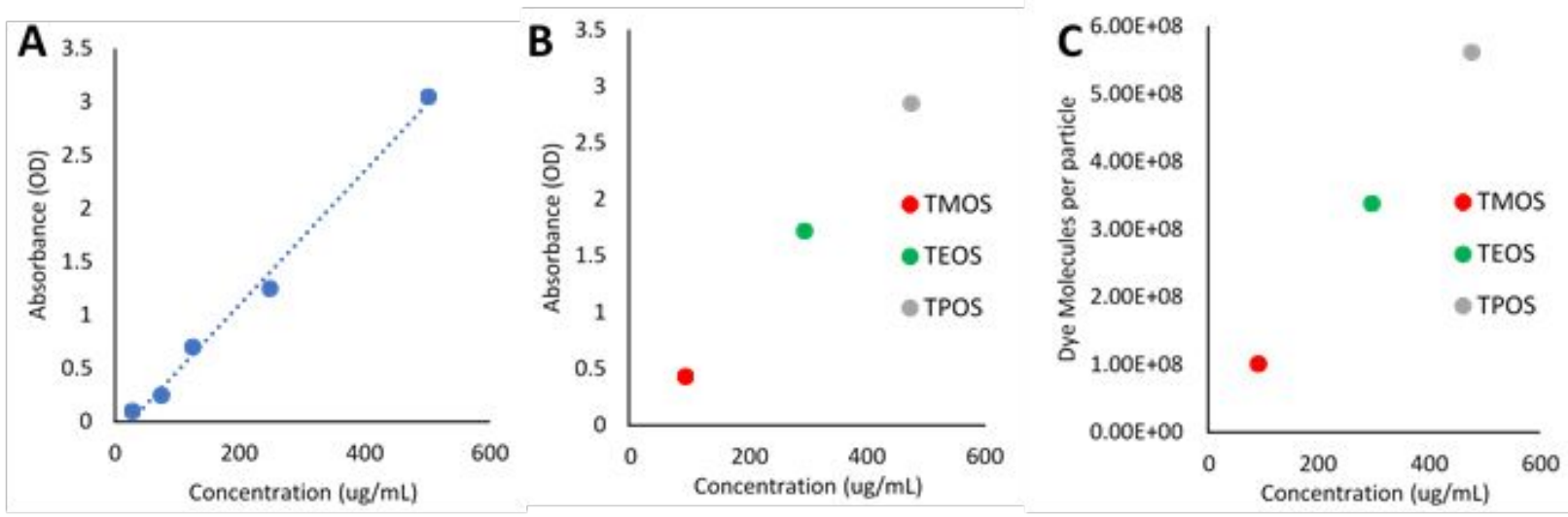

Figure S2 Characterization of IR780 dye within TROS. Graphs depict (A) IR780 standard curve, (B) measurement of optical density compared to IR780 dye within TROS, and (C) number of dye molecules per particle for given concentrations of IR780 in TROS. 

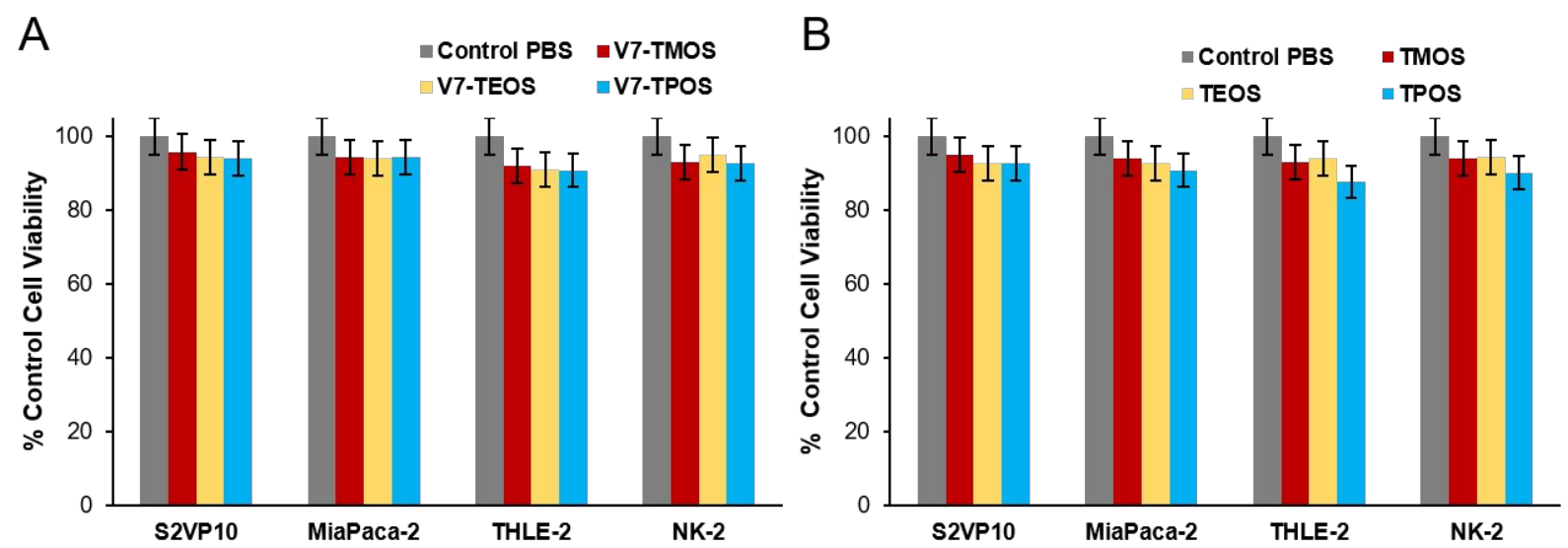

Figure S3 Evaluation of cell viability following TROS and V7-TROS treatment. (A) No significant toxicity was observed in malignant (S2VP10 and MiaPaca-2) or non-malignant (THLE-2 and NK-2) cells at any particle size for V7 actively targeted TROS ( $p>0.05)$. (B) No significant toxicity was observed in malignant (S2VP10 and MiaPaca-2) or non-malignant (THLE-2 and NK-2) cells at any particle size for passively targeted TROS p >0.05. 


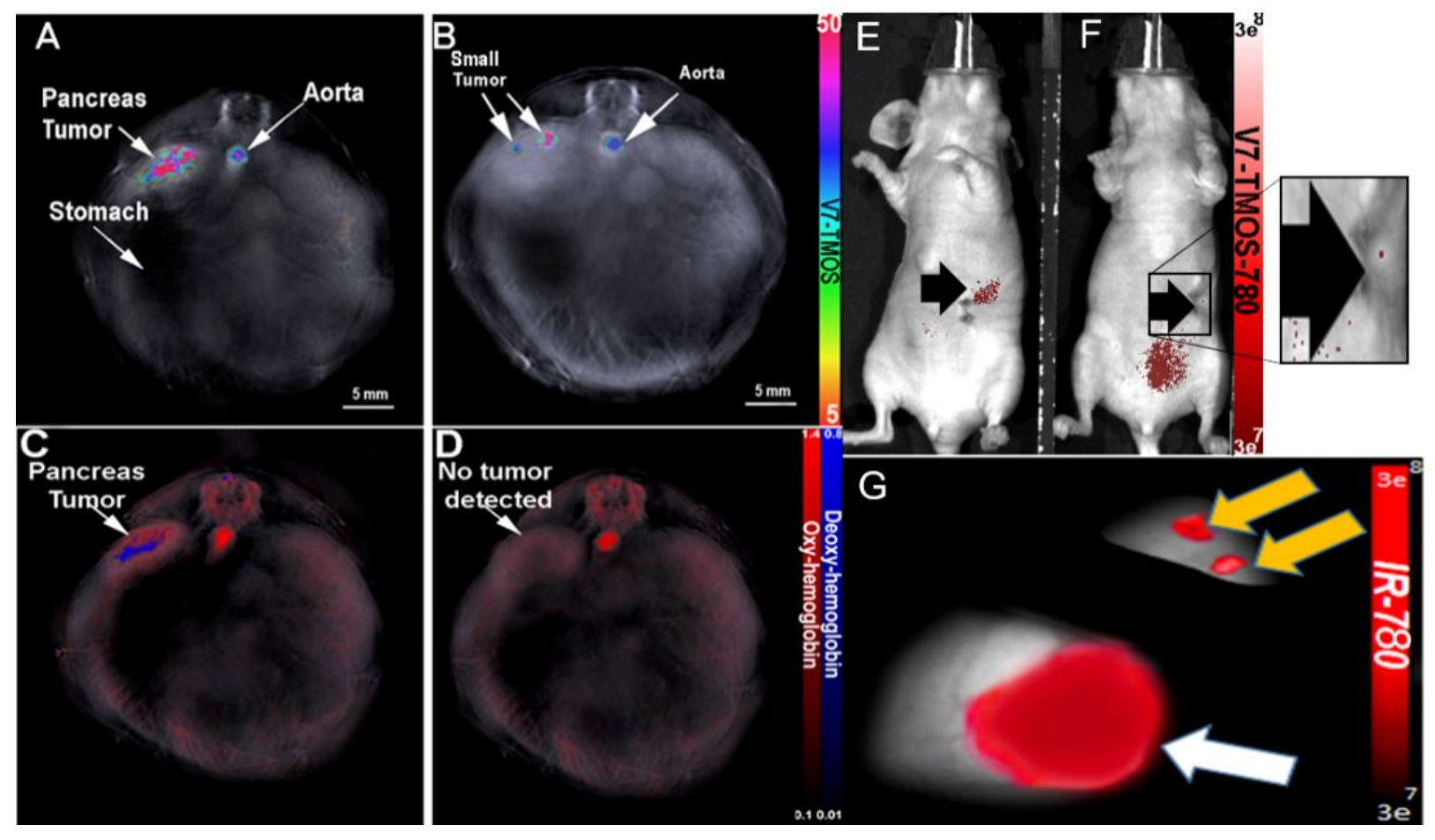

Figure S4 MSOT detection of sub-millimeter PDAC tumor via V7-TMOS in vivo. Orthotopic sub-millimeter $(0.72 \mathrm{~mm})$ PDAC tumor detected using V7-TMOS (A) or oxy/deoxyhemoglobin (C). Orthotopic sub-millimeter $(0.23 \mathrm{~mm})$ PDAC tumor detected with V7-TMOS (B). The $0.23 \mathrm{~mm}$ tumor was not detected with oxy/deoxyhemoglobin only (D). NIR fluorescence was used to detect sub-millimeter tumors of size $0.72 \mathrm{~mm}$ (E). NIR fluorescence was unable to detect PDAC tumors of $0.23 \mathrm{~mm}$ (F). Confirmation of submillimeter tumor using NIR fluorescence(G). 


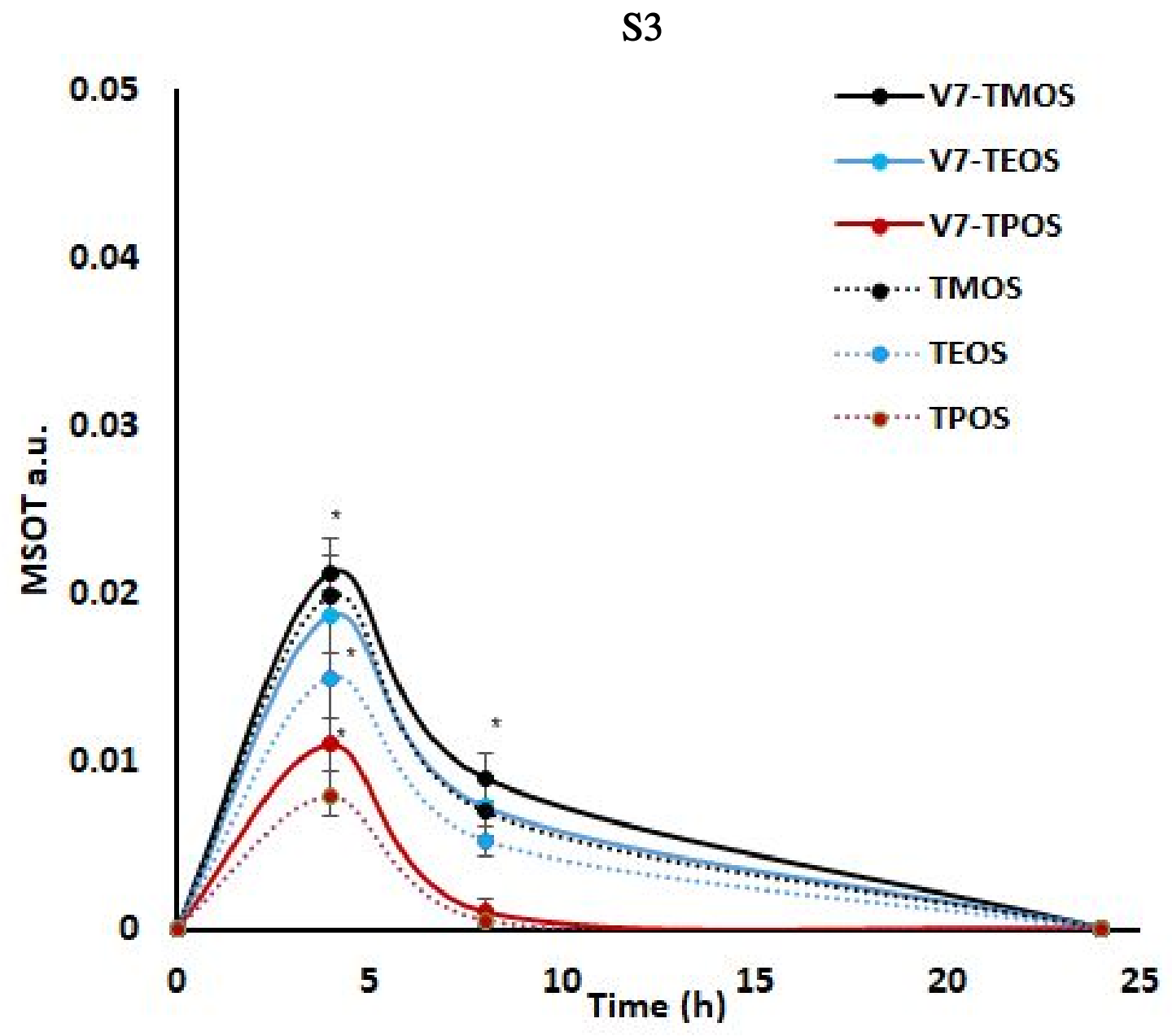

Figure S5 Assessment of blood retention of V7-TROS and TROS signal at timepoints of 0, 4h,

$8 \mathrm{~h}$, and $24 \mathrm{~h}$ post intravenous injection. Signal was measured using a region of interest analysis at a single snapshot at each timepoint in the aorta. The levels of V7-TMOS and V7-TEOS in the 
blood (represented by the aorta) were not statistically different $(\mathrm{p}=0.57)$, however both V7-

TMOS and V7-TEOS were significantly higher than V7-TPOS $(\mathrm{p}=0.046)$.

S4 